DE DE GRUYTER

OPEN

G

BULGARIAN ACADEMY OF SCIENCES

CYBERNETICS AND INFORMATION TECHNOLOGIES • Volume 15, No 5

Special Issue on Control in Transportation Systems

Sofia • 2015

Print ISSN: 1311-9702; Online ISSN: 1314-4081

DOI: 10.1515/cait-2015-0015

\title{
Bi-Level Optimization in a Transport Network
}

\author{
Todor Stoilov ${ }^{1}$, Krasimira Stoilova ${ }^{1}$, Markos Papageorgiou ${ }^{2}$, \\ Ioannis Papamichail ${ }^{2}$ \\ ${ }^{1}$ Institute of Information and Communication Technologies, Bulgarian Academy of Sciences, Sofia \\ 1113 \\ ${ }^{2}$ Dynamic Systems and Simulation Laboratory, Technical University of Crete, Chania, Greece \\ E-mails: todor@hsi.iccs.bas.bg k.stoilova@hsi.iccs.bas.bg markos@dssl.tuc.gr, ipapa@dssl.tuc.gr
}

Abstract: This paper applies a bi-level formalism for the optimal control of an urban transportation network. The well known store-and-forward model in traffic control is utilized in order to increase the control space of the optimization problem. Mainly, the store-and-forward models apply the split as a control argument, assuming the traffic light cycle as a constant parameter. The paper shows that by using a bi-level formalism the control problem can be defined within increased control space comprising both the split and the cycle. Both are found as optimal solutions of a bi-level optimization problem.

Keywords: Traffic control, multilevel systems, bi-level optimization, computer systems, autonomic computing.

\section{Introduction}

The aim of the control of transportation networks is to keep the network capacity on its nominal level. The capacity of the traffic network can be considerably deteriorated when congestion appears on the network links. Thus, the control objective of the traffic control is to restrict the events due to the congested traffic conditions like oversaturation and spillback of the waiting vehicles on the links. The current traffic control approaches usually focus on either urban or freeway traffic. For the urban areas, the traffic lights are the main control actuators used. The freeway traffic is usually controlled, applying ramp metering policies [9].

This paper addresses the control policies applied for the urban areas. An urban network comprises streets, interconnected with junctions. The basic principle of the traffic flow control in the urban areas is minimization of the lost times, the passage times, and the number of stops during driving. All these criteria are functions of the 
queue lengths, arising on the links. The control of the urban traffic focuses on the estimation of the delays and queue lengths, which result from the traffic control at the junctions. Thus, the traffic delays and queues participate in the control criteria of the optimization problems. As the store-and-forward model is of primary consideration in the urban control, a short description is given in the next section.

\section{Overview of the store-and-forward model}

The research and development of the urban signal control particularly address saturated traffic conditions. The store-and-forward modeling allows the development of efficient optimization control solutions. Store-and-forward modeling of the traffic networks has been suggested at first by G a z is and P o t t s [6]. This model formalizes in a simple way the flow process in an urban network. Due to its internal simplicity, it is applied for the cases of large scale congested networks. Some authors have developed traffic control strategies of urban areas, applying store-and-forward model [2, 3, 8, 12]. The most important variables are the queue lengths $x_{i}$ (or simply vehicles) expressed by the number of vehicles on link $i$. The drawback of this model origins from the constraint control space that it can apply. Particularly, only split optimization can be performed while the time cycle and offsets must be derived by other control problems. The graphical presentation of the store-and-forward model is given in Fig. 1. The traffic demands and exit flows are categorized into 4 types, based on their generation and destination.

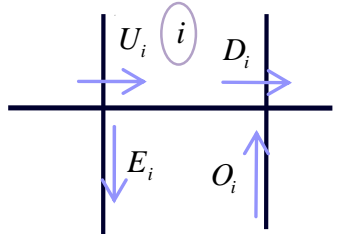

Fig. 1. Graphical presentation of the store-and-forward model

Using the notation $N_{i}(t)$ for the number of vehicles on link $i$ at time $t$, this number is formalized as

$$
N_{i}(t)=A_{i}(t)-L_{i}(t),
$$

where $A_{i}(t)$ denotes the arriving vehicles at link $i$ at time $t ; L_{i}(t)$ - leaving vehicles at link $i$ at time $t$.

Using the notation from Fig. 1, it follows:

$$
\begin{aligned}
& A_{i}(t)=U_{i}(t)+O_{i}(t), \\
& L_{i}(t)=D_{i}(t)+E_{i}(t),
\end{aligned}
$$

where $O_{i}(t)$ denotes the vehicles that originate from link $i$; $E_{i}(t)$ - vehicles that ended within link $i$; $U_{i}(t)$ - vehicles which enter in link $i$ through the junction; $D_{i}(t)$ - vehicles that exit from link $i$ to the next junction.

Relation (1) can be expressed in a discrete time form as

$$
x_{i}(k+1)=x_{i}(k)+T\left[q_{i}(k)-e_{i}(k)+d_{i}(k)-o_{i}(k)\right],
$$

where: $x_{i}(k+1), x_{i}(k)$ are vehicles in link $i$ for the time period $[k T,(k+1) T], T$ is the 
control interval; $q_{i}(k)$ - the input flow $(\mathrm{veh} / \mathrm{h}), \quad q_{i}=\frac{U_{i}}{T} ; o_{i}(k)-$ the outflow (veh/h), $o_{i}=\frac{D_{i}}{T} ; e_{i}(k)-$ exit flow $(\mathrm{veh} / \mathrm{h}), e_{i}=\frac{E_{i}}{T} ; d_{i}(k)-$ demand flow (veh/h), $d_{i}=\frac{O_{i}}{T}$.

The current practice applies the values of $d_{i}$ and $s_{i}$ as known parameters for the control problem. They are estimated like percentage of the input flow $q_{i}(k)$. The control argument is introduced for the outflow as

$$
u_{i}(k)=\frac{s_{i} G_{i}}{c}=s_{i} g_{i} \mathrm{veh} / \mathrm{h},
$$

where $s_{i}$ is the saturation flow of link $i(\mathrm{veh} / \mathrm{h}), c$ is the traffic light cycle (s), $G_{i}$ is the duration of the green light (s), $g_{i}$ is the relative duration of the green light.

A particular advantage of the store-and-forward modelling is that it is applicable to large scale congested networks. The main disadvantage is that due to the particular modeling simplifications, it is applicable only for split optimization, while the cycle time and offsets must be delivered by other algorithms [1].

\section{Optimization criteria for urban traffic control}

The most suited control objective under congested traffic condition is to minimize the events of oversaturation and spillback of the link queues. Thus, an appropriate optimization criterion is the minimization of the relative occupancy of the link $i$ or $x_{i} / x_{i \max }$, where $x_{i \max }$ is the maximum number of vehicles which the capacity of the link can accommodate. This criterion is reasonable from a control point of view and it is formalized as minimization of the total queue lengths $\sum_{i \in \text { network }} x_{i}$ over the urban network [10].

An alternative criterion, which is widely applied now, is the minimization of the Total Time Spent (TTS) by the vehicles of the network. This criterion leads also to the minimization of the sum of $x_{i}$ because its formalization is given like [9]

$$
\mathrm{TTS}=T \sum_{k=1}^{K} N(k) \text {, }
$$

where: $K$ denotes the time horizon; $N(k)$ - total number of vehicles in the network at time $k ; T$ - time interval.

The number of vehicles in the urban network for each control step changes recursively

$$
N(k+1)=N(k)+T[A(k)-L(k)]=N(0)+T \sum_{\kappa=0}^{k}[A(k)-L(k)],
$$

where $N(0)$ are the initial vehicles in the network, $A(k)$ - arriving vehicles at each link (veh/h), $L(k)$ - leaving vehicles at each link (veh/h). 
Hence,

$$
\mathrm{TTS}=\mathrm{TKN}(0)+T^{2} \sum_{k=1}^{K} \sum_{k=0}^{k-1} A(k)-T^{2} \sum_{k=1}^{K} \sum_{k=0}^{k-1} L(k) .
$$

If it is assumed that the arriving vehicles in the network do not depend on the control policy, it is regarded as noise to the control system. Thus, the minimization of TTS is equivalent to the maximization of a weighed sum of the leaving cars from the network, i.e. the maximization of the early exit flows.

Later, in the bi-level control problem, we are going to introduce simultaneously these two criteria for control: to minimize the sum of the queue lengths $x$ and maximize the leaving vehicles $L$ from the network.

\section{Control and optimization approaches}

The store-and-forward modeling of the urban transportation networks is implemented by several control approaches. A feedback control is applied by the linear quadratic optimal control [1]. The split is found like a linear multivariable feedback regulator given as

$$
q(k)=q^{N}-L x(k),
$$

where $q^{N}$ is a fixed control plan, calculated on the basis of historical demands, $L$ is the solution of the corresponding Riccati equation, resulting from the LQ optimization problem, $x(k)$ are the measured vehicles on the links.

Due to the LQ control formulation, this strategy does not allow inclusion of inequality constraints to restrict the queue lengths and the split (green light duration). The control space of this problem concerns only the split of the traffic lights. The cycle and offset are not considered in this problem.

An extension for elaboration of the feedback control is the definition of a quadratic programming problem. It comprises the inequality constraints but its solution leads to an open loop control system. Respectively, such a control strategy needs more on-line computational power to solve the optimization problem. Modifications in nonlinear formalization of the control are given in [1, 3, 4]; with hierarchical decomposition of the control problem [7]; model predictive control [2]; application of the time delay formalism [11]. All these control strategies address the split as a control variable. Attempts to extend the control space are done by [5]. But the cycle and split controls are implemented as independent control algorithms. Such formalization does not allow tackling the internal relations between the control influences, addressing the split duration and the time cycle in a common optimization problem.

\section{Bi-level approach for extension of the control space}

The idea of bi-level control strategy concerns the solution of two optimization problems, which are interconnected (Fig. 2). 


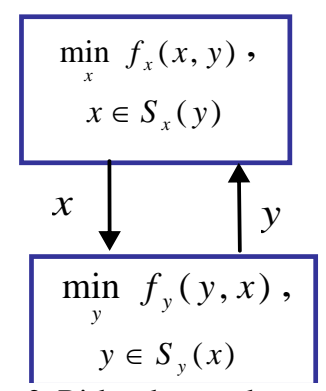

Fig. 2. Bi-level control strategy

The upper level problem assumes the values of $y=y^{*}$ as known parameters and finds an optimal solution $x^{*}(y)$ by solving the problem

$$
\begin{gathered}
\min _{x} f_{x}\left(x, y^{*}\right), \\
x \in S_{x}\left(y^{*}\right) .
\end{gathered}
$$

The solution $x^{*}(y)$ is a function of the parameter $y$. Respectively, the lower level problem assumes $x=x^{*}$ as known parameters and finds its solution $y^{*}(x)$ as a function of $x$.

These two interconnected optimization problems give the solution of the global problem

$$
\begin{gathered}
\min _{x} f_{x}(x, y), \\
x \in S_{x}(y), \\
y \in \arg \left\{\begin{array}{c}
\min _{y} f_{y}(y, x) \\
y \in S_{y}(x)
\end{array}\right\},
\end{gathered}
$$

which means $x^{\text {opt }}$ is the solution of the optimization problem where $y$ modifies the goal function $f_{x}(x, y)$ and the admissible area $S_{x}(y)$. Also, $y$ is a solution of the low level problem, influenced on its turn by $x$.

We will make a comparison of the bi-level optimization problem (2) with a classical optimization problem

$$
\begin{gathered}
\min _{x} f_{x}(x), \\
x \in S_{x} .
\end{gathered}
$$

The bi-level formalization in (2) extends the space of the arguments from $x^{\text {opt }}$ to $\left(x^{\mathrm{opt}}, y^{\mathrm{opt}}\right)$. The bi-level problem optimizes not only the goal function $f_{x}(x, y)$, but an additional one $f_{y}(x, y)$ as well, which means that the optimization goals are increased nevertheless that the function $f_{y}(x, y)$ is subordinated to the main goal $f_{x}(x, y)$. For the classical case (3) the goal function is only one $-f_{x}(x)$. The third peculiarity is that the area of constraints in (2) is considerably wider in comparison with (3), which gives advantages to the bi-level optimization to tackle more constraints in comparison with the classical problem (3). Starting from this conceptual framework, the urban control policy can be complicated to deal with both the split and the cycle as control arguments. 
The paper is developing a bi-level control problem for the case of urban traffic, integrating two optimization problems, Fig. 3. The lower level problem optimizes the queue lengths of the vehicles by changing the splits of the green lights. Their problem assumes that the time cycles are known parameters. The upper optimization problem optimizes the time cycles, assuming given splits. The goal function of this problem is defined as a maximization of the leaving traffic from the urban network. Such a control policy will define in an optimal way both traffic arguments: splits and time cycles. The optimization will address minimization of the queue lengths and maximization of the leaving vehicles which goals are targeted by the control policies, discussed in the previous section.

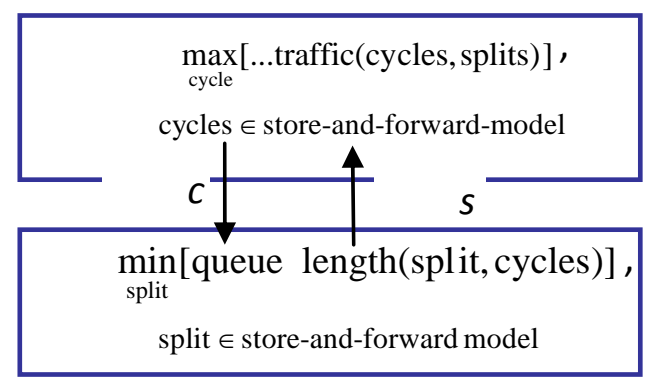

Fig. 3. Traffic control optimization problems

\section{Development of a formal model of the bi-level optimization}

The paper studies the case of an urban network with a structure, given in Fig.4.

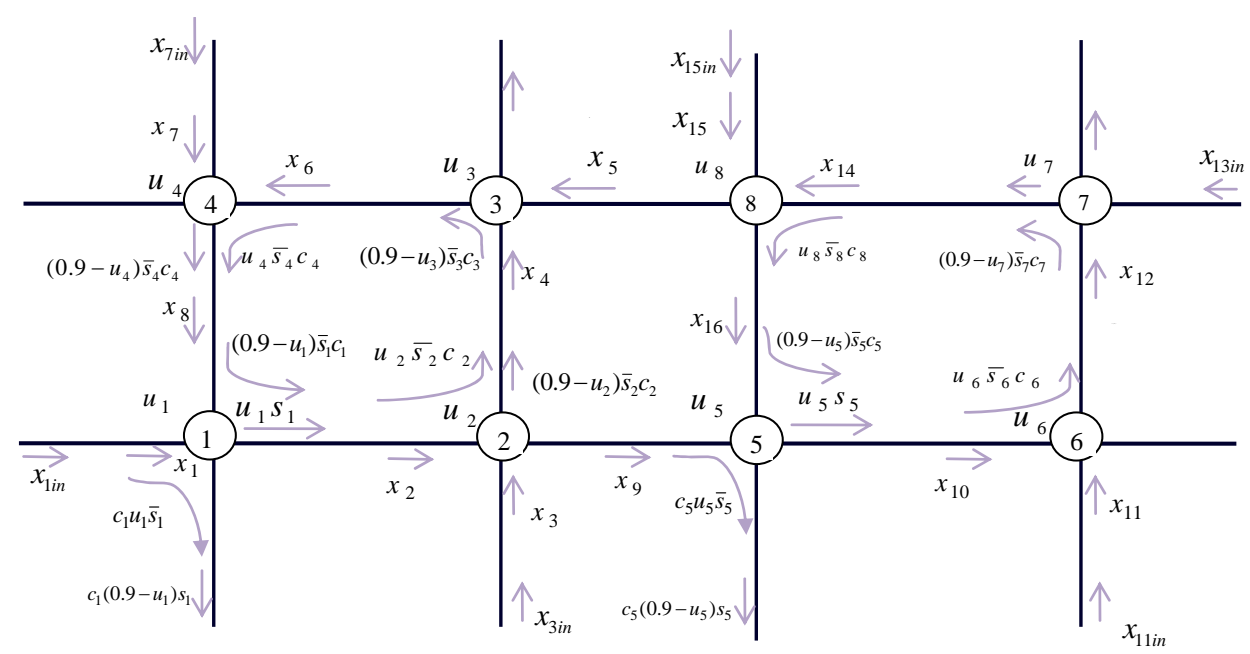

Fig. 4. Traffic network structure

The urban network has been chosen with eight junctions, $j=1, .$. , 8. Each junction is controlled by traffic lights. The split of the green lights in the horizontal 
direction are denoted by $u_{j}$. The amber light is assumed as 0.1 part of the traffic cycle $c_{j}$. Hence, the relation

$$
\text { split(green) + split(red)+split(amber) }=1,
$$

is noted like

$$
u_{j} c_{j}+\text { split }_{j}(\text { red }) c_{j}+0.1 c_{j}=c_{j}
$$

or

$$
\text { split }_{j} \text { (red) }=0.9 c_{j}-u_{j} c_{j}, \quad j=1, \ldots, 8 .
$$

Thus, the split of the red light is not an independent control variable and it is derived simply from the cycle and the split of green of the corresponding junction. The bi-level optimization problem will contain the control space formed by $c_{j}$ and $u_{j}, j=1, \ldots, 8$.

The low level optimization problem is defined in a common way to minimize the queue lengths $x_{i}, i=1, \ldots, 16$, satisfying the conservation equations. Each $x_{i}$ formalizes the vehicles on a corresponding link of the network. In Fig. 5 the conservation equations are illustrated for the first 4 junctions of the network. The following assumption is made in the figure: the saturation flows per junction are the same for the horizontal and the vertical directions. The notations $s_{i}, i=1, \ldots, 4$, are the saturation flows, assumed like known parameters of the control problem. $\bar{s}_{i}, i=1, \ldots, 4$, are coefficients, which take into consideration the amount of vehicles which make a turn and the corresponding saturation of the infrastructure. These values $-s_{i}, \bar{s}_{i}, i=1, . ., 4$, are assumed to be known from statistical measurements or can be changed for each optimization problem from real measurements.

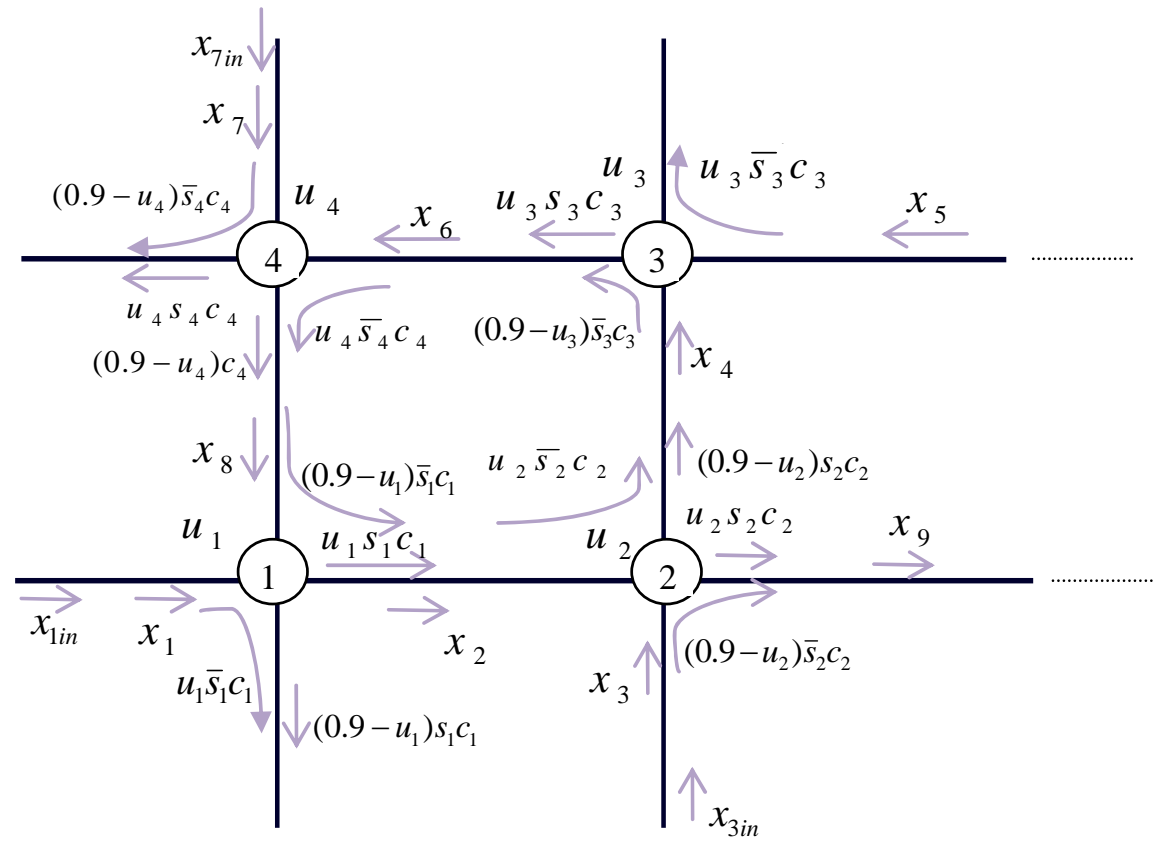

Fig. 5. Arterial road traffic flow 
$x_{i, i n}, i=1, \ldots, 4$, are the demands, which have to enter the urban network. They are assumed as known values needed by the off-line estimations or real time measurements.

The conservation equation for the queues $x_{1}$ and $x_{2}$ are defined like:

$$
\begin{gathered}
x_{1}(k+1) \leq x_{10}(k)+x_{1 \mathrm{in}}(k)-c_{1}(k) u_{1}(k) s_{1}-c_{1}(k) u_{1}(k) \bar{s}_{1}, \\
x_{2}(k+1) \leq x_{20}(k)+c_{1}(k) u_{1}(k) s_{1}+\left[0.9 c(k)-c_{1}(k) u_{1}(k)\right] \bar{s}_{1}-c_{2}(k) u_{2}(k) s_{2}-c_{2}(k) u_{2}(k) \bar{s}_{2} .
\end{gathered}
$$

The inequality forms of the conservation equations give more flexibility because a part of the vehicles can have destination stops on the appropriate link and will not participate in the exiting flow of the link. The low level optimization problem assumes the cycles $c_{i}(k), \quad i=1, \ldots, 4$, as given parameters (not as problem arguments). The low level optimization problem is defined and solved repetitively for each control step to cope with the stochastic nature of changes of the demands and the turning rates $\bar{s}_{i}$. Thus, the discrete type optimization applies a time horizon $k=1$ and the low level optimization becomes a type of mathematical programming.

Following the above considerations, the low level optimization problem becomes a linear-quadratic one in the form

$$
\begin{gathered}
\min _{u, x} f_{x}(x, u), \\
x, u \in S_{x, u},
\end{gathered}
$$

where $x, u \geq 0$,

The goal function is chosen in a quadratic form

$$
f_{x}(x, u)=x^{\mathrm{T}} Q_{1} x+u^{\mathrm{T}} Q_{2} u,
$$

$Q_{1}$ and $Q_{2}$ can be identity or weighted matrices. The engineering meaning of this goal function is the minimization of the total sum of the queues and the constraining of the split duration; $S_{x, u}$ denotes a set of inequalities:

$$
\begin{gathered}
x_{1} \leq x_{10}+x_{1 \mathrm{in}}-c_{1} u_{1} s_{1}-c_{1} u_{1} \bar{s}_{1}, \\
x_{2} \leq x_{20}+c_{1} u_{1} s_{1}+\left(0.9-u_{1}\right) c_{1} \bar{s}_{1}-c_{2} u_{2} s_{2}-c_{2} u_{2} \bar{s}_{2}, \\
x_{3} \leq x_{30}+x_{3 \mathrm{in}}-\left(0.9-u_{2}\right) c_{2} s_{2}-\left(0.9-u_{2}\right) c_{2} \bar{s}_{2}, \\
x_{4} \leq x_{40}+c_{2} u_{2} \bar{s}_{2}+\left(0.9-u_{2}\right) c_{2} s_{2}-\left(0.9-u_{3}\right) c_{3} \bar{s}_{3}-\left(0.9-u_{3}\right) c_{3} s_{3}, \\
x_{5} \leq x_{50}+c_{8} u_{8} s_{8}+\left(0.9-u_{8}\right) c_{8} \bar{s}_{8}-c_{3} u_{3} s_{3}-c_{3} u_{3} \bar{s}_{3}, \\
x_{6} \leq x_{60}+c_{3} u_{3} s_{3}+\left(0.9-u_{3}\right) c_{3} \bar{s}_{3}-c_{4} u_{4} s_{4}-c_{4} u_{4} \bar{s}_{4}, \\
x_{7} \leq x_{70}+x_{7 \mathrm{in}}-\left(0.9-u_{4}\right) c_{4} s_{4}-\left(0.9-u_{4}\right) c_{4} \bar{s}_{4}, \\
x_{8} \leq x_{80}+c_{4} u_{4} \bar{s}_{4}+\left(0.9-u_{4}\right) c_{4} s_{4}-\left(0.9-u_{1}\right) c_{1} s_{1}-\left(0.9-u_{1}\right) c_{1} \bar{s}_{1}, \\
x_{9} \leq x_{90}+c_{2} u_{2} s_{2}+\left(0.9-u_{2}\right) c_{2} \bar{s}_{2}-c_{5} u_{5} s_{5}-c_{5} u_{5} \bar{s}_{5}, \\
x_{10} \leq x_{100}+c_{5} u_{5} s_{5}+\left(0.9-u_{5}\right) c_{5} \bar{s}_{5}-c_{6} u_{6} s_{6}-c_{6} u_{6} \bar{s}_{6}, \\
x_{11} \leq x_{110}+x_{11 i n}-\left(0.9-u_{6}\right) c_{6} s_{6}-\left(0.9-u_{6}\right) c_{6} \bar{s}_{6}, \\
x_{12} \leq x_{120}+c_{6} u_{6} \bar{s}_{6}+\left(0.9-u_{6}\right) c_{6} s_{6}+c c_{6} u_{6} \bar{s}_{6}-\left(0.9-u_{7}\right) c_{7} s_{7}-\left(0.9-u_{7}\right) c_{7} \bar{s}_{7}, \\
x_{13} \leq x_{130}+x_{13 i n}-c_{7} u_{7} s_{7}-c_{7} u_{7} \bar{s}_{7}, \\
x_{14} \leq x_{140}+c_{7} u_{7} s_{7}+\left(0.9-u_{7}\right) c_{7} \bar{s}_{7}-c_{8} u_{8} s_{8}-c_{8} u_{8} \bar{s}_{8}, \\
x_{15} \leq x_{150}+x_{15 i n}-\left(0.9-u_{8}\right) c_{8} s_{8}-\left(0.9-u_{8}\right) c_{8} \bar{s}_{8}, \\
x_{16} \leq x_{160}+c_{8} u_{8} s_{8}+\left(0.9-u_{8}\right) c_{8} s_{8}-\left(0.9-u_{5}\right) c_{5} s_{5}-\left(0.9-u_{5}\right) c_{5} \bar{s}_{5},
\end{gathered}
$$




$$
\begin{aligned}
& x_{j} \geq 0, \quad j=1, \ldots, 16, \\
& u_{j} \geq 0, \quad j=1, \ldots, 8 .
\end{aligned}
$$

After rearrangement, the set of inequalities is presented in the form $A_{1} x+A_{2} u \leq C$,

where $A_{1}=I_{16 \times 16}$ is an identity matrix.

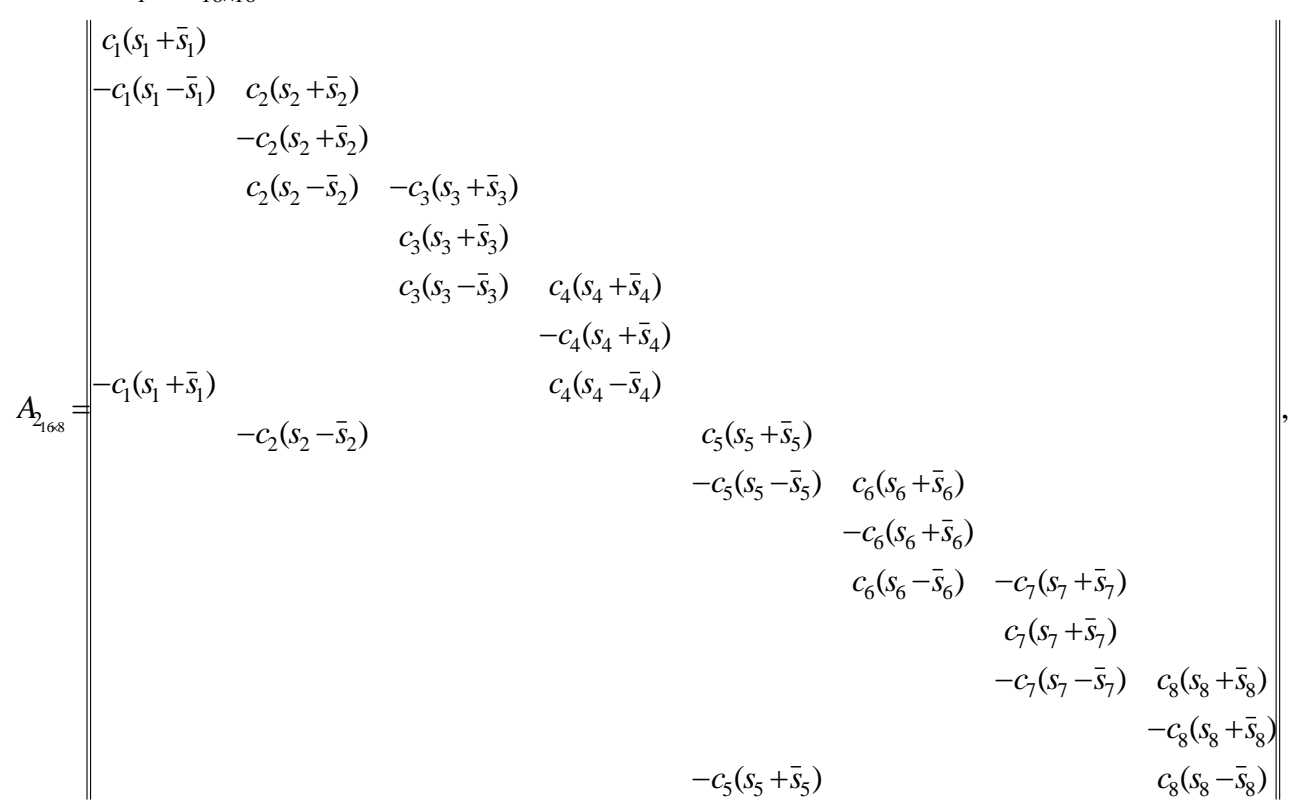

$$
C_{16 \times 1}=\mid \begin{gathered}
x_{10}+x_{\text {lin }} \\
x_{20}+0.9 c_{1} \bar{s}_{1} \\
x_{30}+x_{3 \mathrm{in}}-0.9 c_{2}\left(s_{2}+\bar{s}_{2}\right) \\
x_{40}-0.9 c_{2} s_{2}-0.9 c_{3}\left(s_{3}+\bar{s}_{3}\right) \\
x_{50}+0.9 c_{8} \bar{s}_{8} \\
x_{60}+0.9 c_{3} \bar{s}_{3} \\
x_{70}+x_{7 \mathrm{in}}-0.9 c_{4}\left(s_{4}+\bar{s}_{4}\right) \\
x_{80}+0.9 c_{4} s_{4}-0.9 c_{1}\left(s_{1}+\bar{s}_{1}\right) \\
x_{90}+0.9 c_{2} \bar{s}_{2} \\
x_{100}+0.9 c_{5} \bar{s}_{5} \\
x_{110}+x_{11 \text { in }}-0.9 c_{6}\left(s_{6}+\bar{s}_{6}\right) \\
x_{120}+0.9 c_{6} s_{6}-0.9 c_{7}\left(s_{7}+\bar{s}_{7}\right) \\
x_{130}+x_{13 i n} \\
x_{140}+0.9 c_{7} \bar{s}_{7} \\
x_{150}+x_{15 i n}-0.9 c_{8}\left(s_{8}+\bar{s}_{8}\right) \\
x_{160}+0.9 c_{8} s_{8}-0.9 c_{5}\left(s_{5}+\bar{s}_{5}\right)
\end{gathered} \| .
$$




\section{Upper level optimization problem}

The upper level optimization problem targets the maximization of the vehicles which leave the urban network. The leaving flows, tackled by the problem, are according to Fig. 6.

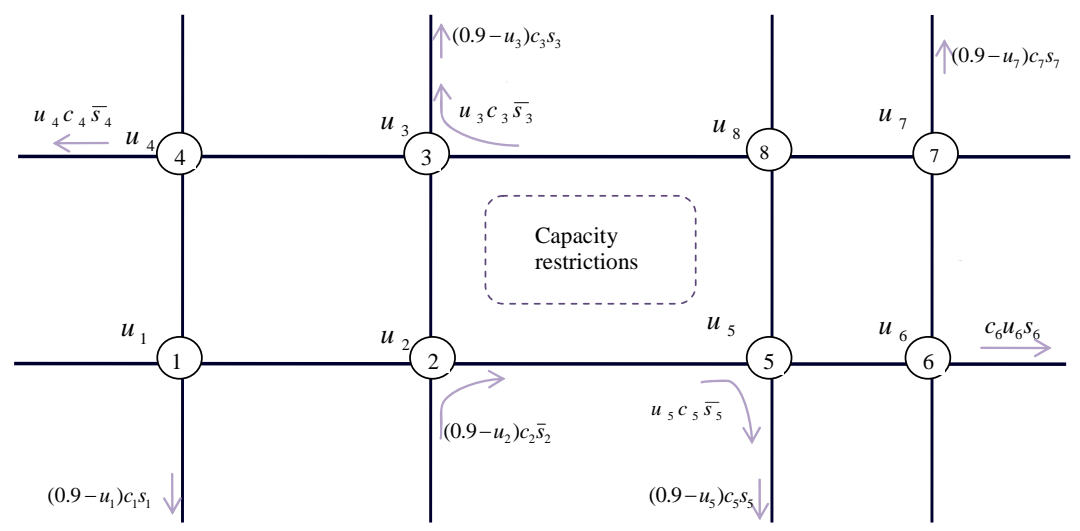

Fig. 6. Network leaving flows

All the other flows are not taken into consideration because they do not decrease the number of the vehicles into the network. The central part of the network endures capacity restrictions. This reason is considered by additional constraints to the upper optimization problem. The capacity constraints are aided also to all internal links of the network. The formal description of the upper level problem takes the form

where $c=\left\|c_{j}\right\|, \quad j=1, \ldots, 8$,

$$
\begin{gathered}
\max _{c}\left(c^{\mathrm{T}} Q_{c} c\right) \\
A_{c} c \leq C_{c},
\end{gathered}
$$

$$
Q_{c}=\operatorname{diag}\left\|\left(0.9-u_{1}\right) s_{1} \quad 1 \quad u_{3}\left(\bar{s}_{3}-s_{3}\right)+0.9 s_{3} \quad u_{4} s_{4} \quad u_{5}\left(\bar{s}_{5}-s_{5}\right)+0.9 s_{5} \quad u_{6} s_{6} \quad\left(0.9-u_{7}\right) s_{7} \quad 1\right\| .
$$

The goal function targets the maximization of the flows which leave the network of Fig. 1. Several flows which go round the network do not enter the network like $c_{1} u_{1} \bar{s}_{1},\left(0.9-u_{6}\right) c_{6} \bar{s}_{6}$. The last flows are not taken into consideration of the goal matrix $Q_{c}$

Because the cycles $c_{2}$ and $c_{8}$ do not influence directly the leaving flows, their coefficients into the goal function could have constant values. For the current case they are assumed equal to 1 .

The feasible area of the problem is defined from considerations of constraining the internal flows according to the capacity restrictions

$$
\begin{gathered}
c_{1}\left[u_{1}\left(s_{1}-\bar{s}_{1}\right)+0.9 \bar{s}_{1}\right] \leq L_{1}, \\
c_{2}\left[u_{2}\left(\bar{s}_{2}-s_{2}\right)+0.9 s_{2}\right] \leq L_{2},
\end{gathered}
$$




$$
\begin{gathered}
c_{3}\left[u_{3}\left(s_{3}-\bar{s}_{3}\right)+0.9 \bar{s}_{3}\right] \leq L_{3}, \\
c_{4}\left[u_{4}\left(\bar{s}_{4}-s_{4}\right)+0.9 s_{4}\right] \leq L_{4}, \\
c_{5}\left[u_{5}\left(s_{5}-\bar{s}_{5}\right)+0.9 \bar{s}_{5}\right] \leq L_{5}, \\
c_{6}\left[u_{6}\left(\bar{s}_{6}-s_{6}\right)+0.9 s_{6}\right] \leq L_{6}, \\
c_{7}\left[u_{7}\left(s_{7}-\bar{s} 7\right)+0.9 \bar{s}_{7}\right] \leq L_{7}, \\
c_{8}\left[u_{8}\left(\bar{s}_{8}-s_{8}\right)+0.9 s 8\right] \leq L_{8}, \\
c_{2}\left[u_{2}\left(s_{2}-\bar{s}_{2}\right)+0.9 \bar{s}_{2}\right]+c_{8}\left[u_{8}\left(s_{8}-\bar{s}_{8}\right)+0.9 \bar{s}_{8}\right] \leq L_{9}, \\
c_{\min } \leq c_{j} \leq c_{\max }, \quad j=1, \ldots, 8,
\end{gathered}
$$

where $L_{j}, j=1, \ldots, 9 ; c_{\min }, c_{\max }$ - predefined values for capacity constraints and eligible durations of the cycles.

For definition of the upper level problem, the control engineers have a relative freedom to choose the exact form of the goal function, the form and content of the constraints. For this research the authors try to keep a simple form of the upper level problem in order to direct their attention to the overall bi-level problem behaviour. The goal function has an engineering meaning of (flow) ${ }^{2}$. Analytically, the product $c^{\mathrm{T}} Q_{c}^{\mathrm{T}}$ represents a flow. To provide (flow) ${ }^{2}$, the relation $c^{\mathrm{T}} Q_{c}^{\mathrm{T}} Q_{c} c$ takes place. From numerical considerations, the matrix $Q_{c}^{\mathrm{T}} Q_{c}$ can be substituted simply by $Q_{c}$, which does not change the engineering meaning of (flow) ${ }^{2}$ of the goal function. Particularly, the presented upper problem applies a goal function in the form $\left(c^{\mathrm{T}} Q_{c} c\right)$ which misses the linear part for simplification of the problem. The goal function can be modified if additional considerations have to be taken for the control problem. In the same way, the interconnected constraints can describe additional relations between the flow behaviours into the urban network.

For the case of exploration of the bi-level formal problem this paper has restricted additional considerations taken into account. The upper level problem assumes the splits $u_{i}, i=1, \ldots, 8$, as known parameters and the problem arguments are the cycles $c_{j}, j=1, \ldots, 8$.

\section{Numerical simulations of the bi-level control problem}

The problem has been solved repetitively. Starting with initial values of the vehicles $x_{i 0}, i=1, \ldots, 16$ in the network, the problem is solved in the sequence low level problem - upper level problem, till finding convergence to the global solutions. After that, the obtained values $x_{i}^{\text {opt }}$ are assumed as new initial values $x_{i 0}=x_{i}^{\text {opt }}, i=1, \ldots, 16$, and the new bi-level problem is solved again. The numerical results from the simulation have been compared with the classical open loop linear quadratic control. Having in mind the low level optimization problem, it gives an exact LQ optimal solution for the case when the cycles $c_{j}, j=1, \ldots, n$, are fixed. If the cycles $c_{j}$ are found according to the upper level optimization and taking into consideration that the upper and low level problems are interconnected, the bi-level problem will calculate other solutions, which differ from the classical LQ 
optimization. To compare these two control policies (LQ and bi-level), two criteria are introduced:

- the total amount of the queues at each link for the time duration of the simulation period;

- the total amount of vehicles, which leave the urban network for the time of simulation.

These two integral criteria were calculated for each step of the numerical simulation. The integration is done for all links and it is different for each time step. It is concluded that the bi-level control problem results in fewer amounts of vehicle queues and a bigger number of vehicles which leave the urban network, Figs 7 and 8. This means that the bi-level policy has a potential for better servicing of the users in crossing the urban area.

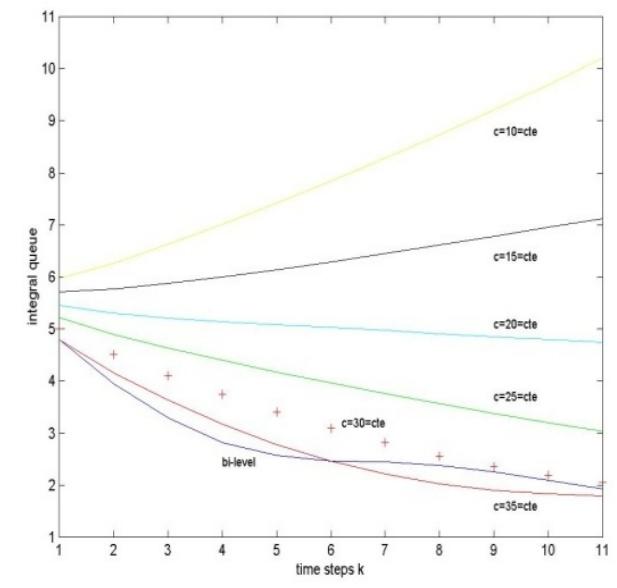

Fig. 7. Total amount of the queues

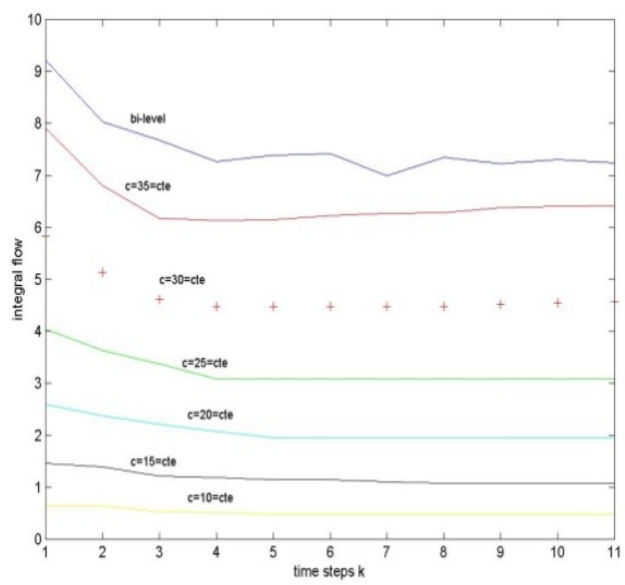

Fig. 8. Total amount of outgoing flows

\section{Conclusions}

The paper applies a new formal description of the control policy on an urban transportation area. A bi-level optimization problem is defined and numerically tested. The optimization problem applies the widely used model of store-andforwarding phenomenon. The bi-level formalism achieves and increases the control space not only of the splits, but in the common problem it includes the time cycles. The classical LQ formalization applies the time cycles as constant values and thus, the control space contains only the split. Having a wider control space, it is possible to achieve more and additional goals in the control process and to consider more control constraints. The current research applies optimization towards decrease of the total queues and maximization of the vehicles which cross the urban area. The next steps for exploration of the bi-level models and optimization imply evaluation of the computational power, needed for real time implementation of the bi-level control policy. 
Acknowledgement: This work is partially supported by the project "AComIn: Advanced Computing for Innovation" grant 316087 funded by the European Commission in FP7 Capacity (2012-2016) and COST TU1102.

\section{References}

1. A b oudolas, K., M. Papag e orgiou, E. Kos mat op oula s. Store and Forward Base Methods for the Signal Control Problem in Large-Scale Congested Urban Road Networks. Transportation Research, Part C: Emerging Technologies, Vol. 17, 2009, pp. 163-174.

2. B er g, M., A. H e g y i, B. de S c hut t e r, H. He ll e n d o o r n. Integrated Traffic Control for Mixed Urban and Free Way Networks: A Model Predictive Control Approach. - EJTIR, Vol. 7, 2007, No 3, pp. 223-250.

3. D i a ka ki, C., M. P a p a g e org i o u, K. A b o ud o las. A Multivariable Regulator Approach to Traffic-Responsive Network - Wide Signal Control. - Control Engineering Practice, Vol. 10, 2002, No 2, pp. 183-195.

4. Diakaki, C., V. D in op oulou, K. A boudolas, M. Papageorgiou. Signal Management in Real Time for Urban Traffic Networks. Technical Report, Technical University of Crete, IST Programme, 2002.

5. Diakaki, C., V. Dinopoulou, K. Aboudolas, M. Papag e orgiou, E. Shabat, E. S e ider, A. L e ib o v. Extension and New Applications of the Traffic Signal Control Strategy TUC. - In: Transportation Research Record. No 1856 2003, pp. 202-211.

6. Gazis, D., R. P otts. The Oversaturated Intersection. - In: Proc. of 2nd International Symposium on Traffic Theory, London, UK, 1963, 221-237.

7. H o m o l o v a, J. Hierarchical Regulator of Traffic Flows. Traffic Flow Control. http://library.utia.cas.cz/prace/20050107.pdf

8. K a s h a n i, H. R., G. N. S a r i d i s. Intelligent Control for Urban Traffic Systems. - Automatica, Vol. 19, 1983, No 2, pp. 191-197.

9. P a p a g e org i o u, M., A. K o t s i a l o s. Freeway Ramp Metering; An Overview. - In: IEEE Intelligent Transportation Systems. Conference Proceedings, 2000, pp. 228-239.

10. P a p a g e org i u, M., C. D i a ka ki, V. D i n o p o u l o u, A. K o t s i a los, Y. W an g. Review of Road Traffic Optimal Control Strategies. - Proceedings of IEEE, Vol. 91, 2003, pp. 2043-2067.

11. Tamura, H. Decentralized Optimization for Distributed Lag Models of Discrete Systems. Automatica, Vol. 11, 1975, pp. 593-602

12. Y in, H., S. C. W o n g, J. X u, C. K. W o n g. Urban Traffic Flow Prediction Using a Fuzzy Neural Approach. - Transportation Research, Part C, Vol. 10, 2002, pp. 85-98. 